\title{
International Healthcare Management
}

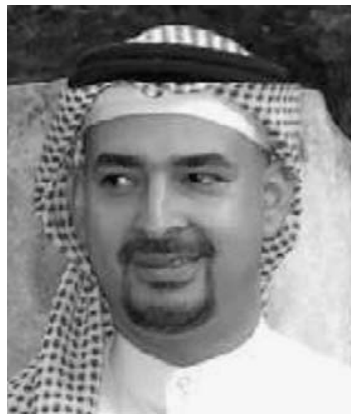

Dr. Waleed Alansari, MBA

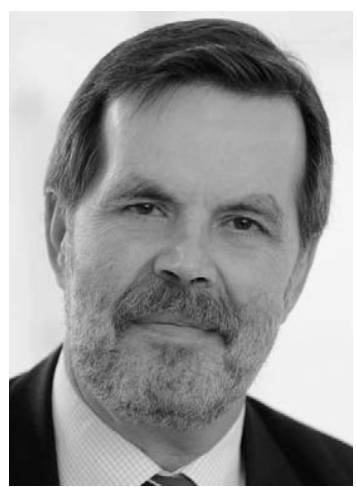

Prof. Dr. Jochen Breinlinger O'Railly

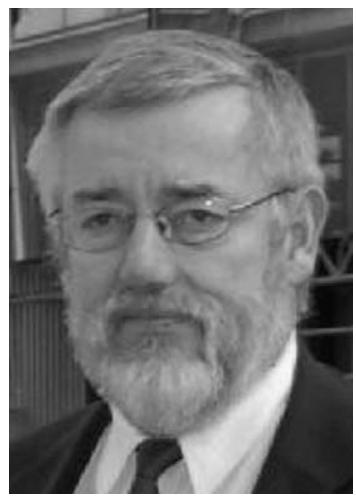

Prof. Dr. Jens-Uwe Niehoff

\section{Bibliografie}

Dol http://dx.doi.org/ 10.1055/s-0031-1273773 Gesundheitswesen 2011; 73: 121-123

(c) Georg Thieme Verlag KG Stuttgart · New York ISSN 0941-3790

Korrespondenzadresse

Prof. Dr. med. habil. J.-U. Niehoff

Healthcare Management Consulting

jniehoff@gmx.net
Die 5. deutsch-chinesische Konferenz zum Management öffentlicher Aufgaben 2010 hat einen maßgeblichen Schwerpunkt auf das Themenfeld Healthcare Management gelegt. Die Hochschule für Wirtschaft und Recht Berlin bietet hierzu seit vielen Jahren erfolgreich Curricula national und international an. Nachfolgend werden aus einer der Schlüsselreden dieser Konferenz auszugsweise Positionen zum Thema International Healthcare Management zusammengefasst.

Es kann geschätzt werden, dass derzeit etwa 50 Staaten aktiv für die Inanspruchnahme ihrer nationalen medizinischen Versorgungseinrichtungen werben. Darunter sind Staaten, deren eigene Gesundheitssysteme im Aufbau sind, die selbst Gesundheitsdienstleistungen im Ausland finanzieren oder erhebliche Probleme haben, eine ausreichende Anzahl von Fachkräften aus dem Potenzial des eigenen Landes zu entwickeln.

Entsprechend einer Richtlinie der EU, die für 2011 erwartet wird, soll ab 2013 innerhalb der EU die freie Arztwahl für ambulante und stationäre Leistungen möglich werden. Wir sind sicher, diese Öffnung wird in ihren Folgen nicht allein von dem aktiven Entscheidungsverhalten der Patienten, wohl aber von den strategischen Erwägungen maßgeblicher Akteure auf der Versorgungs- wie der Finanzierungsseite bestimmt werden.

Hierbei sei betont, dass der tatsächliche Raum für eine internationalisierte Krankenversorgung im Bezugsraum der EU eher eine kleine Region abdeckt.

Diese Internationalisierung oder Globalisierung hat sehr unterschiedliche Ursachen und verfolgt ebenso verschiedene Interessen. Entsprechend weit gefächert, auch kontrovers, sind die Bewertungen. Eine grenzüberschreitende Krankenversorgung ist allerdings für viele Regionen der Erde längst Realität und auch keine wirklich neue historische Erfahrung. Das gilt zumindest für privat finanzierte (damit auch privilegierte) Leistungen oder für solche, die von dritten Parteien aus unterschiedlichen Gründen finanziert werden.

Die globale Bedeutung dieser Entwicklungen ist offensichtlich und macht das Management von Gesundheitsversorgung zum Gegenstand internationaler Aktivitäten. Gegenwärtig spiegeln sich diese in vielen Namen; wie „Medical Tourism“, „Medical Travel“, „Health Tourism“, „International Healthcare“, „Global Healthcare“, „Cross-Border Healthcare“ oder "Health Service Outsourcing“. Jeder dieser Begriffe assoziiert, nicht nur in Deutschland, unterschiedliche Reaktionen und
Bewertungen. In jedem Fall aber stehen alle diese Namensgebungen für eine Internationalisierung des Versorgungsmanagements, der eine entsprechende Professionalisierung, gestützt auf Forschung und Lehre, bislang eher randständig gegenüber steht.

Wenn diese Beschreibung richtig ist, sollte das International Healthcare Management zwingend ein eigenständiges Feld für Forschung, Lehre und Praxis des Managements der Krankenversorgung werden und zwar ebenfalls international und hoffentlich auch getragen von der Kooperation einer entsprechenden „International Scientific Community“, die zu entwickeln zunächst auch eine nationale Aufgabe ist oder sein sollte.

Eine große Zahl global agierender Investoren und Investmentgesellschaften ist in der Gesundheitswirtschaft aktiv. Die Aktivitätsfelder beziehen sich auf Investitionen und den Betrieb von (zumeist) Krankenhäusern. Kooperationen mit staatlichen oder öffentlich-rechtlichen Partnern sind die Regel, nicht die Ausnahme.

Folgt man der International Standard Industry Classification (ISIC), dann schließt die Gesundheitsindustrie die Gesundheitsversorgung, die Produktion und Distribution von Geräten, Ausrüstungen und Dienstleistungen, selbstverständlich Forschung, Lehre und Ausbildung ein. All dies eröffnet nicht nur künftig, sondern ist bereits heute ein riesiges Feld für kompetentes Management. Die Frage, die sich tatsächliche oder potenzielle Partner stellen müssen, kann nicht lauten, ob eine solche Einschätzung richtig ist oder nicht. Die zu beantwortende Frage ist allein, ob es ein Interesse gibt, an dieser Entwicklung Anteil haben zu wollen oder nicht. Hier ist selbstverständlich die nächste Frage, wer jeweils diese Frage stellt und sie, getragen von welchem Interesse, beantwortet. Nur eines wird in jedem Fall richtig sein: Bildung wird ohne spezifisch ausgerichtete Forschung nicht erfolgreich oder wirksam werden, und sie verlangt Plattformen des Dialoges, national wie international. Es ist gleichgültig, ob dies den internationalen wirtschaftlichen Aktivitäten vorausgeht oder nachfolgt. Es bedarf des Willens zur internationalen Zusammenarbeit auf diesem Gebiet und der Etablierung tragfähiger akademischer Strukturen.

Es gibt viele gute Gründe, das globale Healthcare Management auch in seinen nationalen Perspektiven zu einem dauerhaften Diskussionsgegenstand mit entsprechend

1. interessierten und international profilierten (oder an einer solchen Profilierung interessierten) Universitäten, 
2. an einem solchen Dialog interessierten wirtschaftlichen Akteuren der Gesundheitswirtschaft und

3. Akteuren der finanziellen Sicherungssysteme für die Gesundheitsversorgung zu machen.

Wir bezweifeln die Notwendigkeit einer eigenen Konferenz- und Tagungsindustrie. Wir sind jedoch überzeugt, dass konkrete $\mathrm{Zu}-$ sammenarbeit und die Stärkung der jeweils nationalen Ressourcen und Strukturen eine unbedingt notwendige Begleitung für die Globalisierung der Gesundheitsversorgung und ihres Managements sein muss.

In unserer Sicht ist das International Healthcare Management in seinen Kernelementen schnell beschrieben: Es soll den Zugang zu und die Prozesse der Gesundheitsversorgung kranker Menschen, bzw. von Menschen, die auf professionelle Hilfe und Unterstützung angewiesen sind, organisieren und absichern. Dies kann durch die unmittelbare Krankenversorgung ebenso geschehen, wie durch die Unterstützung und die Begleitung der Planung, Konstruktion, Ausrüstung und den Betrieb von Einrichtungen der Gesundheitsdienstleistungen.

International Healthcare Management schließt also den Transfer von

- Erfahrung und Kompetenz,

- Information,

- Medizinprodukten,

- Fachfrauen und -männern der Gesundheitsversorgung,

- Erfahrungen und Konzepten der Ausbildung und Forschung im Themenfeld Versorgungsmanagement,

- humanitärer Unterstützung und Hilfe,

- grenzüberschreitender Krankenversorgung (durch die Mobilität von Patienten, von Professionen und/oder die Kommunikation) und

- Kapital

ein.

Wir alle sind Zeugen eines Prozesses, der medizinische Versorgungskapazitäten im globalen Maßstab und auch im massivem (Kosten-) Wettbewerb erschließt. Zugleich existiert hier ein (offenbar attraktives) Feld für Investitionen von Staaten, non-profit und von for-profit Organisationen, die einen nennenswerten Anteil der jeweils nationalen Ökonomien darstellen.

In Bezug auf diese Entwicklungen gibt es viele kontroverse Diskussionen. Dabei sind die empirischen Kenntnisse zu diesen Entwicklungen eher gering oder wenig verbreitet. Wer sich aus einer internationalen Perspektive mit diesen Entwicklungen auseinandersetzt, wird kaum an der Einschätzung vorbeikommen, dass - sieht man von den Exporten der „Versorgungsindustrie" und der Inanspruchnahme von medizinischen Dienstleistungen in Deutschland durch „Medizintouristen“ ab - die Bundesrepublik eine eher geringe Rolle im „International Healthcare Management“, auch auf dem Gebiet von Forschung und Bildung spielt.

Wir sind der Auffassung, dass zentrale Probleme dringend einer fokussierten Forschung, auch mit dem Ziel des Transfers der Methoden und Ergebnisse, bedürfen. Das Management in diesem Bereich internationaler Aktivitäten hat sich mit sehr spezifischen Problemen auseinanderzusetzen. Es sollen hierzu nur Beispiele gegeben werden:

- Akkreditierungsstandards,

- nationale Leitsätze des Sozial-, Gesundheits- und Haftungsrechtes,

- Regulation der Finanzierung und Abrechnung von Gesundheitsdienstleistungen,
- das Management einer grenzüberschreitenden Behandlung von Patienten, ihrer Vorbereitung und ihrer nachsorgenden Betreuung,

- das Management von Informationen und Datensicherheit und

- die Wahrnehmung und der Umgang mit Erwartungen der Patienten vor dem Hintergrund national spezifischer kultureller Erfahrungen und religiöser Bindungen.

Healthcare Management Kompetenz ist mehr als Buchhaltung oder als die Verwirrung über die Erwägungen von Selma Muskin oder Michael Grossman über den finanziellen Grenznutzen öffentlicher Aufwendungen für die Gesundheitsvorsorge oder die von Kenneth Arrow über die Inhomogenität der Versorgungsbedarfe im Fall von Krankheit. Das Management der Versorgung kranker Menschen braucht fundamentale Einsichten in die sehr spezifischen Bedingungen dieser humanen Dienstleistung, deren maßgebliche Qualitätskriterien sich um die Interpretation von medizinischer Notwendigkeit, angemessener Intervention, Ziel-Mittel-Relationen (Effektivität) und Ziel-Aufwands-Relation (Effizienz), inter- und intraprofessionelle Kooperation und Verantwortung gruppieren.

Ein besonderes Problem des International Healthcare Management sind die Akkreditierung von Dienstleistern und die systematische Abbildung der Varianz der Dienstleistungsergebnisse. Die Lösung dieser beiden Probleme darf als zentral unterstellt werden, zumal effektive Lösungen nur jeweils im nationalen Rechtsrahmen und unter entsprechender hoheitlicher Aufsicht möglich sein dürften. Man wird - nebenher - gespannt sein dürfen, ob und ggf. wie sich die Europäische Union mit diesem Problem auseinandersetzt, bzw. welchen Rechtsrahmen sie hier normativ für das International Healthcare Management vorgibt. Das gilt nicht nur, aber in besonderer Weise für das spezifische Segment von Angeboten der sog. experimentellen Therapien, die globale Versorgungsangebote sehr schnell zu einem gefährlichen, in jedem Fall auch teuren Abenteuertourismus machen können. Das ist sicherlich ein Problem höchster Priorität und zwar zum Schutz der Patienten wie auch des Dienstleistungssektors. Die Lizenzierung, die Akkreditierung, die Konsentierung von Mindeststandards und vor allem die Transparenz verlangen hohe Standards, die von Anbeginn im Fokus globaler Versorgungsangebote stehen müssen.

Die Herausbildung eines global verfügbaren Angebots der Gesundheitsversorgung, bedarf internationaler, mindestens aber explizit auf der Vertragsebene formulierter Regeln. Diese Entwicklungen werden spürbar die öffentlichen und ggf. die staatlichen Versorgungseinrichtungen, die Anforderungen an die Steuerung der Versorgungsprozesse sowie deren Supervision beeinflussen. Wir sind davon überzeugt, dass auch das Professionsfeld des International Healthcare Management sich weiter und zwar über das Attribut „international“ hinaus, spezialisieren wird. Beispiele könnten sein:

- das Management der Inanspruchnahme von Leistungen gemäß der jeweiligen Rechtsbedingungen und Versorgungsverträge,

- das spezifische Management der Entgeltung von Leistungen, bzw. das Management der jeweiligen methodischen Implikationen für den (Fehl-)Anreiz zur Risikoselektion,

- das Management von funktions- und ergebnisorientierten Leistungen von Versorgungsteams, spezifisch in der Krankenversorgung, der Rehabilitation und der Pflege,

- das Management von Versorgungseinrichtungen sowie ihrer personalen, finanziellen und technischen Ressourcen und 
- die Planung, Ausrüstung, vertragliche Ausgestaltung und der Betrieb von Versorgungseinrichtungen, z. B. Krankenhäusern, sowie die Refinanzierung der Investitionen.

Wenn sich das Healthcare Management internationalisiert oder globalisiert, bedarf es eines sehr präzisen Verständnisses des jeweiligen nationalen Gesundheitssystems. Das ist nicht nur, aber eben auch ein Sprachproblem.

Die wachsende Bedeutung globaler Mechanismen der Gesundheitsversorgung und der Internationalisierung ihres Managements, wirft die Frage nach universellen Merkmalen eines Berufs-, Lehr- und Forschungsfeldes "International Healthcare Management" auf.

Es sollte in dieser Beziehung der folgende Standard konsensfähig sein und in entsprechenden Forschungs- und Lehrkonzeptionen beachtet werden können

- Grundlagen von Public Health,

- Internationale Systeme der Finanzierung der Krankenversorgung und jeweils deren spezifischen Regulationsmethoden und -wirkungen,

- nationale rechtliche Standards der Akkreditierung, Supervision und der Haftungen im Falle der Krankenversorgung,

- die Kenntnis der wesentlichen Fall- und Personenklassifikationen, die als Produktklassifikationen medizinischer Versorgung international praktiziert werden,

- Methoden und rechtliche Regelungen zum Produktmarketing in der medizinischen Versorgung,

- Ethik des Managements von Krankenversorgung,
- die organisatorischen und funktionalen Strukturmuster von international üblichen Versorgungseinrichtungen,

- das Qualitäts- und Prozessmanagement in Gesundheitseinrichtungen,

- Grundlagen der Makro- und Mikroökonomie der Gesundheitsversorgung und deren jeweils spezifische nationale Umsetzungskonzepte (Wettbewerb, Planung, Priorisierungsmodelle, explizite Rationierungsmethoden, gesundheitsökonomische Studientypen),

- Finanzierung, Bilanzierung, Wirtschaftsplanung, Controlling und Risikobewertung von Modellen der Krankenversorgung und strategisches Investitionsmanagement,

- Personalmanagement,

- Kommunikation, Teambildung, Projektmanagement, Verhandlungsführung,

- Geschäftsplanung,

- Management von Informationen und Informationstechnologien, Innovationsmanagement und

- Dienstleistungsmanagement für medizinische Versorgungsprozesse.

Wir sind davon überzeugt, dass der professionelle Manager in der Gesundheitsversorgung von Morgen ein Spezialist sein muss, der die sehr besondere Natur dieses Bereichs humaner Dienstleistungen beherrscht und nicht ohne weitergehende Ausbildung zwischen dieser und anderen Managementqualifikationen in Produktion und Dienstleistung ausgetauscht werden kann. 